\title{
Latest Advances in Imaging Oxidative Stress in Cancer
}

\author{
Hannah E. Greenwood and Timothy H. Witney \\ School of Biomedical Engineering and Imaging Sciences, King's College London, London, United Kingdom
}

Oxidative stress is the imbalance of harmful reactive oxygen species (ROS) and the action of neutralizing antioxidant mechanisms. If left unchecked, the deleterious effects of oxidative stress result in damage to DNA, proteins, and membranes, ultimately leading to cell death. Tumors are highly proliferative and consequently generate high levels of mitochondrial ROS. To compensate for this and maintain redox homeostasis, cancer cells upregulate protective antioxidant pathways, which are further amplified in drug-resistant tumors. This review provides an overview of the latest molecular imaging techniques designed to image oxidative stress in cancer. New probes can now assess heterogeneous ROS and antioxidant production within tumors and across lesions. Together, the noninvasive imaging of these dynamic processes holds great promise for monitoring response to treatment and predicting drug resistance and may provide insight into the metastatic potential of tumors.

Key Words: ROS; oxidative stress; antioxidant; molecular imaging; $\mathrm{MRl}$; PET; fluorescence

J Nucl Med 2021; 62:1506-1510

DOI: $10.2967 /$ jnumed.120.256974

\section{D}

uring their transformation, cancer cells acquire metabolic adaptations that sustain their rapid proliferation, progression, and protection from cell death (1). This metabolic reprogramming provides the basis for the clinical imaging and staging of tumors with ${ }^{18} \mathrm{~F}-\mathrm{FDG}$ PET. The ability to take up glucose and secrete lactate even when oxygen is present (termed aerobic glycolysis) is a key feature of malignancy (2). However, whereas defective mitochondrial respiration was historically thought to accompany aerobic glycolysis, tumors metabolize glucose concurrently through both glycolysis and the tricarboxylic acid cycle at rates far higher than those in healthy tissue (3).

Oxidative stress, the imbalance between harmful reactive oxygen species (ROS) production and the cell's ability to neutralize these reactive intermediates (Fig. 1A), is a common consequence of elevated mitochondrial respiration. Leakage of electrons from complex I and III of the electron transport chain results in the partial reduction of oxygen and the subsequent generation of ROS. These reactive species include hydrogen peroxide $\left(\mathrm{H}_{2} \mathrm{O}_{2}\right)$, singlet oxygen $\left({ }^{1} \mathrm{O}_{2}\right)$, the hydroxyl radical $(\cdot \mathrm{OH})$, peroxides $\left(\mathrm{O}_{2}{ }^{2-}\right)$, and superoxides $\left(\mathrm{O}_{2} \cdot{ }^{-}\right)$. Other subcellular regions of ROS generation

Received May 13, 2021; revision accepted Jul. 15, 2021.

For correspondence or reprints, contact Timothy H. Witney (tim.witney@ kcl.ac.uk).

Published online August 5, 2021.

Immediate Open Access: Creative Commons Attribution 4.0 International License (CC BY) allows users to share and adapt with attribution, excluding materials credited to previous publications. License: https://creativecommons. org/licenses/by/4.0/. Details: http://jnm.snmjournals.org/site/misc/permission. xhtml.

COPYRIGHT (C) 2021 by the Society of Nuclear Medicine and Molecular Imaging. include peroxisomes ( $\beta$-oxidation of fatty acids) and the endoplasmic reticulum (protein oxidation), or as by-products of enzymatic reactions by cyclooxygenases, nicotinamide adenine dinucleotide phosphate oxidases, xanthine oxidases, and lipoxygenases (4). Furthermore, multiple components of the tumor-immune microenvironment, such cancer-associated fibroblasts and myeloid-derived suppressor cells, macrophages, and activated $\mathrm{T}$ cells, provide an exogenous source of ROS (5).

In conjunction with DNA damage, chemotherapy and radiotherapy produce high levels of oxidative stress in tumors (6). If left unchecked, oxidative stress causes damage to DNA, proteins, and lipids and, ultimately, the initiation of cell death. To maintain redox homeostasis and prevent the harmful consequences of oxidative stress, cancer cells upregulate a network of ROS scavenging enzymes and antioxidant pathways (7). As well as generating mitochondrial ROS, cancer metabolism fuels antioxidant production through oxidative pentose phosphate pathway (PPP) generation of reduced nicotinamide adenine dinucleotide phosphate (NADPH) and amino acid metabolism (Fig. 1B). NADPH maintains the antioxidant capacity of thioredoxin reductase and glutathione peroxidase, whereas import of cysteine via system $\mathrm{x}_{\mathrm{C}}{ }^{-}$is required for glutathione biosynthesis, the body's most abundant antioxidant (8). In this review, we describe the exciting recent advances in the field of oxidative stress imaging and their potential applications.

\section{FLUORESCENT PROBES}

Fluorescence-based imaging systems and probes are widely used for the measurement of a broad spectrum of ROS (9). Elevation of ROS above baseline levels (e.g., after therapeutic intervention) is often assumed to be synonymous with oxidative stress, although in reality oxidative stress can only be inferred from their measurement. A common method to detect multiple forms of ROS is the use of a reduced nonfluorescent dye that, once oxidized, produces a fluorescent product (switch-on sensors). Hydrocyanines are a class of fluorescent probes that are produced by reducing the iminium cation of commercially available cyanine dyes with $\mathrm{NaBH}_{4}$. On their oxidation by superoxide and hydroxyl radicals, the original cyanine dye is formed. These dyes fluoresce from 560 to $830 \mathrm{~nm}$ and are ionic impermeable moieties, resulting in their intracellular trapping and the generation of contrast (10). Thiophene-bridged hydrocyanine probes overcome some of the limitations of the first-generation probes, which suffer from high autoxidation, low Stokes shifts, and poor stability. Another widely used switch-on sensor for generalized ROS detection is the CellROX (ThermoFisher Scientific) family of compounds (11).

Mitochondria and the plasma membrane are particularly vulnerable to oxidative damage. If left unchecked, oxidative stress results in lipid peroxidation, which can be measured by BODIPY 581/ $591 \mathrm{C} 11$ (ThermoFisher Scientific). Multiple ROS species can oxidize the polyunsaturated butadienyl substituent, resulting in a shift 
in fluorescent emission from 590 to $510 \mathrm{~nm}$. Changes in lipid ROS can subsequently be quantified by measuring the ratio of red to green fluorescence (11).

\section{Probes for the Selective Imaging of Individual Reactive Species}

In addition to assaying oxidative activity in cells, fluorescent probes have been developed for species-specific ROS detection, including superoxide (dihydroethidium), hydrogen peroxide $\left(2^{\prime}, 7^{\prime}\right.$ dichlorofluorescein), and singlet oxygen (trans-1-(2' -methoxyvinyl)pyrene). Most probes are not truly specific for individual reactive species; rather, they exhibit enhanced selectivity for different ROS. These fluorescent probes rely on varied mechanisms of action to generate contrast. For example, Amplex Red (Thermo Fisher Scientific) is selectively oxidized by hydrogen peroxide in a reaction mediated by horseradish peroxidase. $2^{\prime}, 7^{\prime}$-dichlorofluorescein

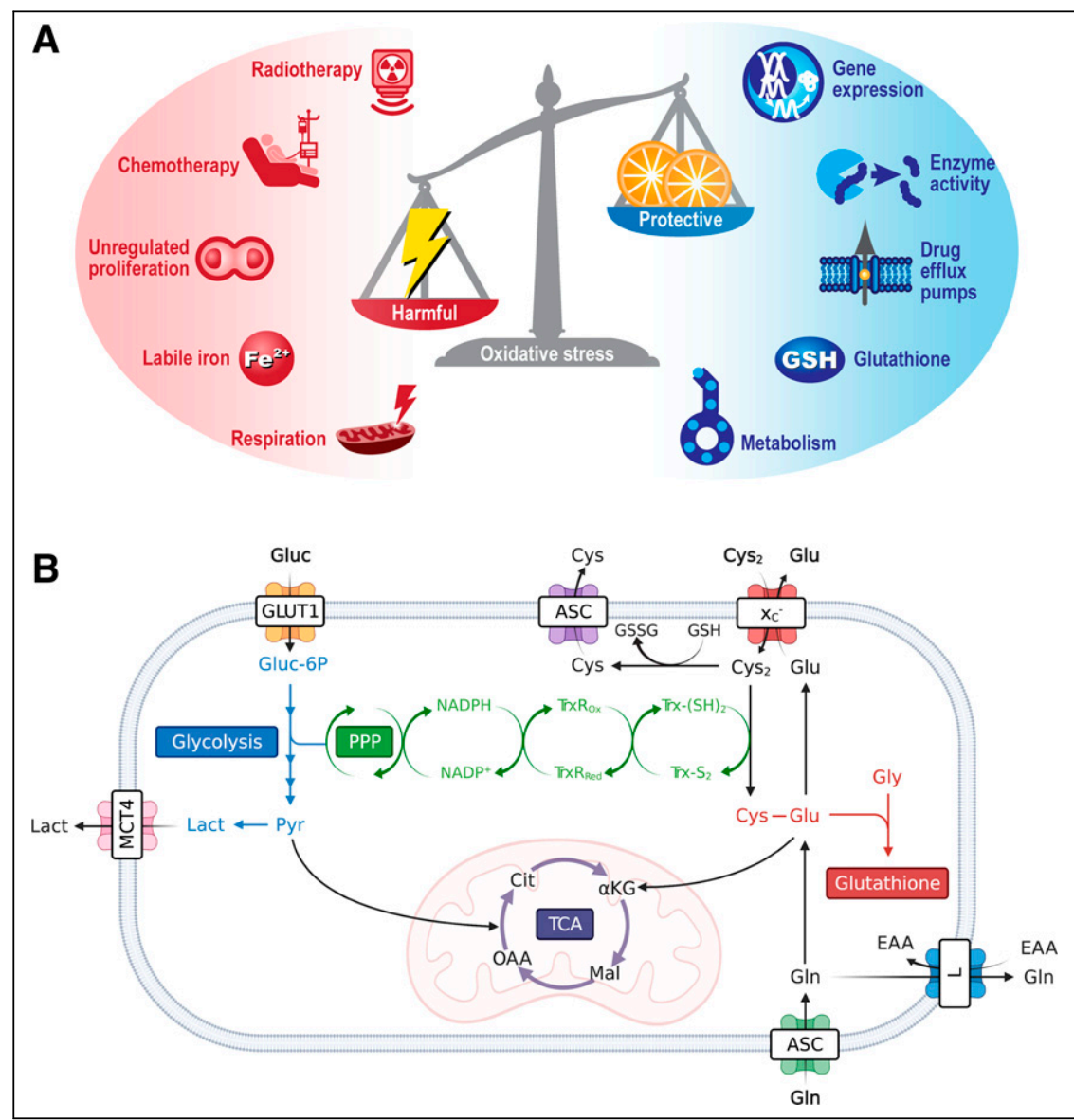

FIGURE 1. Mediators and protective mechanisms that regulate oxidative stress. (A) Oxidative stress is imbalance between harmful ROS and neutralizing antioxidants. ROS can be formed by either intrinsic or extrinsic factors, with a network of intracellular free radical scavenger systems designed to maintain redox homeostasis and protect against cellular damage. (B) Metabolism is key regulator of intracellular antioxidants NADPH, glutathione, and the thioredoxin pathway. For clarity, tricarboxylic acid cycle and glycolysis have been abbreviated. ASC $=$ alanine/serine/cysteine transporter subfamily; $\alpha \mathrm{KG}=\alpha$-ketoglutarate; Cit $=$ citrate; Cys $=$ cysteine; $\mathrm{Cys}_{2}=$ cystine; $\mathrm{EAA}=$ essential amino acids; Glu = glutamate; Gln = glutamine; Gluc = glucose; Gluc-6P = glucose 6-phosphate; GLUT1 = glucose transporter 1; Gly = glycine; GSH = glutathione; GSSG = oxidized glutathione; $\mathrm{L}=$ system $\mathrm{L}$ amino acid transporter; Lact = lactate; Mal = malate; $\mathrm{MCT} 4$ = monocarboxylate transporter 4; NADPH = reduced nicotinamide adenine dinucleotide phosphate; $\mathrm{NADP}^{+}=$ nicotinamide adenine dinucleotide phosphate; $\mathrm{OAA}=$ oxaloacetate; $\mathrm{Pyr}=$ pyruvate; $\mathrm{TCA}=$ tricarboxylic acid cycle; $\operatorname{TrxR}_{\mathrm{ox}}=$ oxidized thioredoxin reductase; $\mathrm{TrxR}_{\mathrm{Red}}=$ reduced thioredoxin reductase; $\operatorname{Trx}-\left(\mathrm{S}_{2}\right)=$ thioredoxin-disulfide reductase; $\operatorname{Trx}-\left(\mathrm{SH}_{2}\right)=$ thioredoxin-dithiol reductase; $\mathrm{x}_{\mathrm{C}}{ }^{-}=$ system $\mathrm{x}_{\mathrm{C}}^{-}$.
$\left(\mathrm{H}_{2} \mathrm{DCF}\right)$ is an alternative dye used for the quantitation of intracellular hydrogen peroxide. In the diacetate form of $2^{\prime}, 7^{\prime}$-dichlorofluoresnonfluorescent probe passively diffuses through the cell cein, resulting in then oxidized by hydrogen peroxide to produce $2^{\prime}, 7^{\prime}$-dichlorodihydrofluorescein, which is highly fluorescent. Mitochondrion-specific eroxides can also be visualized by MitoSOX Red (ThermoFisher , a cationic derivative of dihydroethidium that is electrophoretically taken up into actively respiring mitochondria and fluo-

\section{maging of Glutathione}

As the most abundant thiol-containing antioxidant, glutathione is a surrogate marker of cellular antioxidant capacity. The fluorescent dyes monobromobimane and monochlorobimane readily react with low-molecular-weight thiols, including glutathione, and in doing so form fluorescent adducts. An additional thioltracking dye is ThiolTracker Violet (Thermo Fisher Scientific), which is also retained intracellularly through adduct formation and whose fluorescent signal is 10 times greater than that of bimane compounds (11).

An important consideration is that optical imaging is constrained by overlying tissue both absorbing and scattering the exciting or emitted light. Fluorescence is therefore better suited for cell-based imaging and superficial or intraoperative smallanimal preclinical work than for translational applications.

\section{PET IMAGING}

Systemic oxidative stress has been assessed in the clinic by measuring oxidized proteins, oxidized lipids, and serum antioxidants (12). Although relatively easy to collect and measure, these biomarkers provide no tissue-specific information to better inform any subsequent intervention. Molecular imaging using PET can reveal subtle biologic changes that occur both within tumors and across multiple heterogeneous lesions.

\section{PET Imaging of ROS}

The successful application of fluorescent probes to ROS and antioxidant imaging has resulted in the adaptation of these small molecules to PET, often through the incorporation of ${ }^{18} \mathrm{~F}$. Turn-on mechanisms after radiotracer oxidation, however, cannot be used for the generation of contrast by PET, and alternative methods of intracellular trapping are required. Chu et al. demonstrated the advantages of radiolabeling the fluorescent dye dihydroethidium with ${ }^{18} \mathrm{~F}$ and showed its ability to measure superoxide production after treatment with 
doxorubicin in cells grown in culture. After its oxidation, ${ }^{18} \mathrm{~F}$ labeled dihydroethidium becomes charged and can intercalate DNA, intracellularly trapping the tracer (13). Other fluorescent scaffolds, such as hydrocyanines (14), have also been labeled with ${ }^{18} \mathrm{~F}$ as a method to image oxidative stress in vivo. In addition to ROS-sensing fluorophores, chemiluminescent probes based on luminol have been used for ROS detection. Recently, a galliumlabeled luminol derivative (galuminox) was shown to selectively accumulate in the mitochondria of tumor cells after ROS induction, with ${ }^{68}$ Ga-galuminox selectively retained in a model of lung inflammation (15). A radiolabeled ascorbate derivative, ${ }^{18} \mathrm{~F}-\mathrm{KS} 1$, is also in the early stages of development for ROS imaging (16).

\section{Imaging the Tumor Antioxidant Response}

Given the short-lived nature of ROS, imaging of the durable downstream consequences of this toxic insult may provide a larger detection window with PET. The transmembrane protein system $\mathrm{x}_{\mathrm{C}}{ }^{-}$is a heterodimeric transporter that is placed centrally within the cell's antioxidant system. The role of system $\mathrm{x}_{\mathrm{C}}{ }^{-}$is to exchange the intracellular amino acid glutamate for the extracellular amino acid cystine. After cystine's uptake, it is rapidly reduced to cysteine, the rate-limiting precursor for glutathione biosynthesis, placing system $\mathrm{x}_{\mathrm{C}}{ }^{-}$as a central regulator of antioxidant homeostasis (17). Elevated system $\mathrm{x}_{\mathrm{C}}{ }^{-}$activity has been exploited by PET imaging tracers such as $(4 S)-4-\left(3-{ }^{18} \mathrm{~F}\right.$-fluoropropyl)-L-glutamate $\left({ }^{18} \mathrm{~F}-\mathrm{FSPG}\right)(18),{ }^{18} \mathrm{~F}$-5-fluoro-aminosuberic acid (19), and ${ }^{18} \mathrm{~F}-\mathrm{hGTS} 13$ (20). Tumor retention of ${ }^{18} \mathrm{~F}-\mathrm{FSPG}$ is redoxsensitive, mediated by the concentration gradient of cystine across the plasma membrane. In an animal modal of ovarian cancer, ${ }^{18} \mathrm{~F}$ FSPG tumor retention decreased in proportion to the degree of oxidative stress induced by chemotherapy (Fig. 2A) (21).

A consequence of ROS-induced membrane peroxidation is the intracellular production of reactive aldehydes that if left unchecked result in catastrophic DNA damage. Many cancer cells upregulate aldehyde dehydrogenases in response to this oxidative stress, which mediates aldehyde detoxification (22). The enzymatic activity of aldehyde dehydrogenase 1A1 has recently been quantified with a novel substrate-based radiotracer (23). Using a complementary strategy, Kirby et al. developed ${ }^{18} \mathrm{~F}-\mathrm{NA}_{3} \mathrm{BF}_{3}$ for the imaging of total aldehydic load through radiotracer-aldehyde complex formation (24). Together, these tracers may provide insight into oxidative stress-mediated lipid peroxidation during anticancer therapy.

\section{MRI}

Several paramagnetic MRI contrast agents have been developed to probe the redox balance of cells and tissues. Stable nitroxide free radicals are cell-permeable reporters of intracellular antioxidant availability, undergoing 1-electron transfer reactions to produce hydroxylamines. The single unpaired electron of nitroxides provides $\mathrm{T}_{1}$ contrast, which disappears on their reduction, the rate of which is dependent on ROS-scavenging systems (25). Nitroxide relaxivity, however, is 20 times less than $\mathrm{Gd}^{3+}$, and contrast is quickly lost after administration. Alternative MRI contrast agents based around activatable paramagnetic complexes have subsequently been developed to overcome these limitations. The oxidation state of both $\mathrm{Mn}^{3+/ 2+}(26)$ and $\mathrm{Fe}^{3+/ 2+}$ complexes (27) alters the intrinsic relaxation properties of MRI probes, enabling a noninvasive measure of cellular redox status.

\section{Hyperpolarized Spectroscopic MRI}

Dynamic nuclear polarization is an emerging technique that increases the sensitivity of MR experiments by more than 10,000 times, allowing dynamic imaging of administered ${ }^{13} \mathrm{C}$-labeled substrates and their metabolic products in vivo (28). Flux through the PPP has been estimated using this technique through the conversion of $\mathrm{U}_{-}{ }^{2} \mathrm{H}, \mathrm{U}_{-}{ }^{13} \mathrm{C}$-glucose to the PPP intermediate 6-phosphogluconate (29). PPP metabolic activity is upregulated in cancer, which generates NADPH to maintain the antioxidant capacity of cells. However, the short polarization lifetime of uniformly labeled glucose at relatively low levels $(\sim 15 \%)$, along with overlapping ${ }^{13} \mathrm{C}$ resonances of 6-phosphogluconate and 3phosphoglycerate (a glycolytic intermediate), is a challenge that currently restricts the widespread use of $\mathrm{U}_{-}{ }^{2} \mathrm{H}, \mathrm{U}-{ }^{13} \mathrm{C}$-glucose. Alternatively, hyperpolarized $\left[1-{ }^{13} \mathrm{C}\right]$ dehydroascorbic acid ([1- $\left.\left.{ }^{13} \mathrm{C}\right] \mathrm{DHA}\right)$, the oxidized form of ascorbic acid (vitamin $\mathrm{C}$ ), has been used to probe tumor redox potential (30). After its uptake by the facilitative glucose transporters, hyperpolarized $\left[1-{ }^{13} \mathrm{C}\right] \mathrm{DHA}$ was rapidly converted to $\left[1-{ }^{13} \mathrm{C}\right]$ vitamin $\mathrm{C}$ in lymphoma (30) and prostate tumors (Figs. 2B-2D) (32), the rate of which was determined by the levels of both glutathione and NADPH (31). Despite the promise of $\left[1-{ }^{13} \mathrm{C}\right] \mathrm{DHA}$ to assess total tumor antioxidant capacity, administration of a $10 \mathrm{mg} / \mathrm{kg}$ dose of DHA to tumorbearing mice resulted in transient respiratory arrest and cardiac depression (31). Optimization of dosing regimens and a greater understanding of DHA toxicity are therefore a prerequisite for clinical imaging with $\left[1-{ }^{13} \mathrm{C}\right] \mathrm{DHA}$.

An important consideration for all MRI-based redox probes is the requirement of high mass doses of contrast agent. Given that these agents are frequently either stable radicals or potent radical scavengers, redox-active MRI probes may also perturb the system that they are measuring, possibly accounting for DHA-induced toxicity.

\section{APPLICATIONS AND FUTURE PERSPECTIVES}

As we have illustrated, several well-characterized imaging agents have shown promise for the noninvasive imaging of oxidative stress in animal models of cancer. Given that ROS are typically short-lived (half-life of $10^{-9} \mathrm{~s}$ for $\cdot \mathrm{OH}$ to $10^{-3} \mathrm{~s}$ for $\mathrm{H}_{2} \mathrm{O}_{2}$ (33)) and encompass a variety of different reactive molecules, frequently at low concentrations, imaging ROS dynamics is a challenging proposition. The cellular antioxidant response to these insults persists, however, on a time scale and magnitude that permit its measurement by medical imaging techniques. If clinically adopted, several applications exist for oxidative stress imaging that could impact disease outcomes.

\section{Response Monitoring}

In conjunction with DNA damage, chemotherapies and ionizing radiation produce high levels of oxidative stress in tumors, with cell death induced in those sensitive to treatment (6). Consequently, redox imaging agents have the potential to assess the efficacy of a wide range of therapies that converge with the induction of oxidative stress. In a recent proof-of-principle study, the tumor antioxidant response to doxorubicin was shown to be an earlier maker than both ${ }^{18}$ F-FDG and tumor volume (21). Furthermore, the imaging window for the measurement of tumor antioxidant response is not limited by a temporally unstable marker (e.g., cell death) (34). Additionally, several therapies have been developed whose primary mechanism of action is the induction of lipid ROS and concurrent membrane peroxidation (35). Redox imaging 


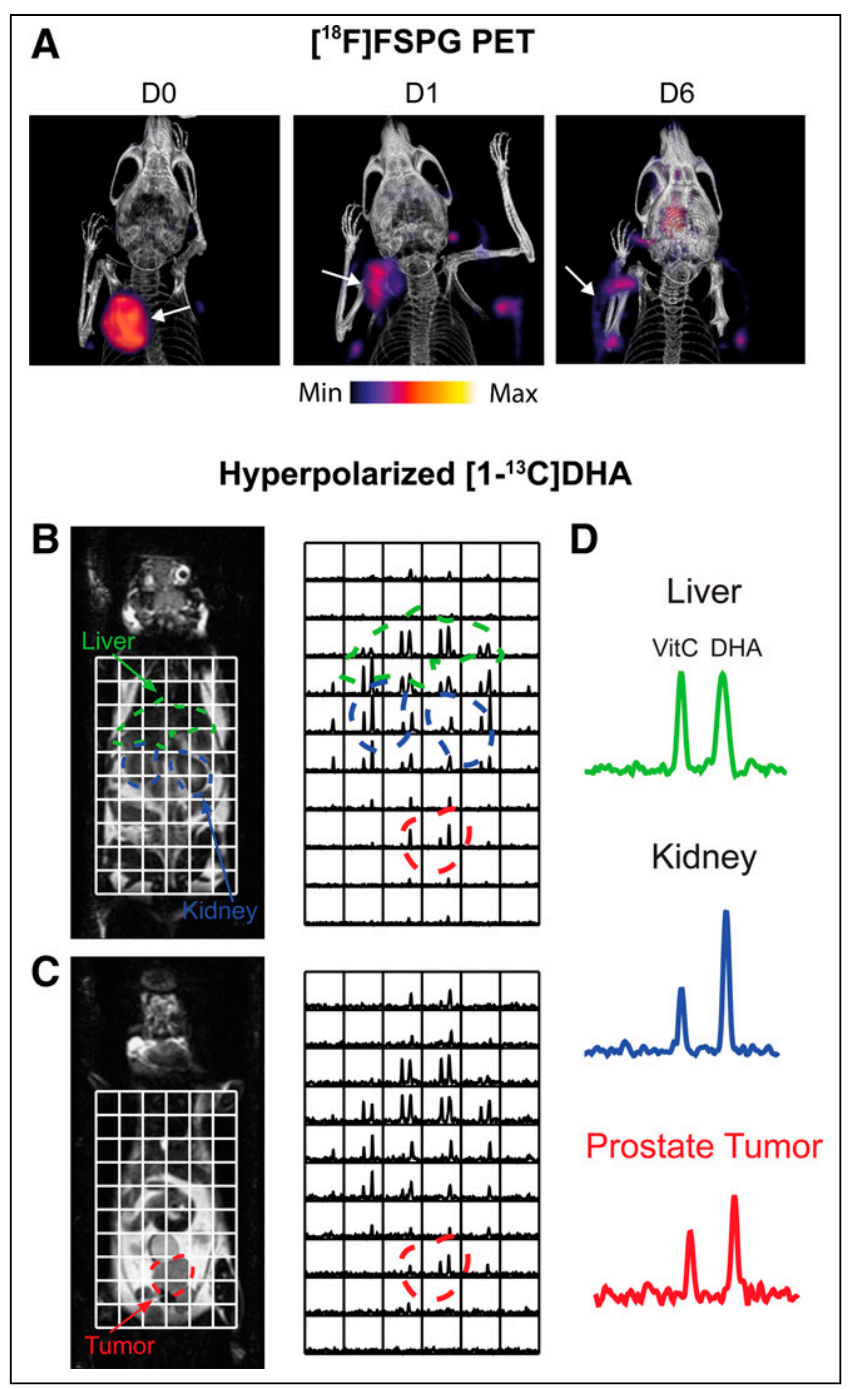

FIGURE 2. Imaging tumor redox status. (A) Changes in ${ }^{18} \mathrm{~F}-\mathrm{FSPG}$ A2780 ovarian tumor retention after oxidizing doxorubicin $\mathrm{HCl}$ liposome (Doxil; ALZA Corp.) therapy. (Reprinted from (21).) (B and C) Sequential coronal T2-weighted images and corresponding ${ }^{13} \mathrm{C}$ 3-dimensional MR spectroscopic images demonstrating distribution of hyperpolarized $\left[1-{ }^{13} \mathrm{C}\right] \mathrm{DHA}$ and vitamin $\mathrm{C}$ in TRAMP (transgenic adenocarcinoma mouse prostate) mouse. Regions of liver, kidney, and prostate tumor are segmented and superimposed on spectral grid (color-coded dashed lines). (D) Representative ${ }^{13} \mathrm{C}$ spectra from liver, kidney, and prostate tumor in TRAMP mouse. D0 = untreated; D1 $=24-\mathrm{h}$ Doxil treatment; D6 $=6 \mathrm{~d}$ after initiation of Doxil treatment. VitC = vitamin C. (Reprinted from (32).)

probes may therefore play an important role in monitoring the response to these novel agents.

\section{Prediction of Drug Resistance}

Elevated antioxidant capacity and the ability to buffer oxidative stress are a hallmark of drug-resistant cancer (36). A noninvasive measure of drug resistance will facilitate early intervention, allowing the clinician to adapt the treatment regimen, with the potential to improve patient outcomes. For widespread utility, the imaging biomarker ideally should be causal to drug resistance, be tumorspecific, result in a positive imaging signal, be generalizable to multiple drugs with different mechanisms of action, have expression that is independent of other factors or conditions, and require a single imaging scan. To date, ${ }^{18} \mathrm{~F}-\mathrm{FSPG}$ imaging of system $\mathrm{x}_{\mathrm{C}}{ }^{-}$ activity has proven to be a good surrogate marker of drug resistance in animal models of ovarian cancer, reporting on the elevated glutathione found in these tumors (37). Further work, however, is needed to determine whether ${ }^{18} \mathrm{~F}-\mathrm{FSPG}$ is a robust marker of drug resistance for multiple cancer types with discrete driver mutations.

\section{Metastases}

Tumor cells experience substantial oxidative stress when they detach from the extracellular matrix and enter the circulation. Anoikis, a form of programmed cell death after loss of anchorage, frequently follows intravasation and restricts the metastatic capabilities of tumor cells (38). The oxidative environment of the bloodstream further limits metastatic efficiency. In anoikisresistant cells, PPP-generated NADPH mitigates the ROS that accompanies loss of attachment to permit cell survival (39). Suppressing oxidative stress by increasing endogenous and exogenous antioxidant availability in vivo further promotes metastasis in multiple models of cancer (1). Consequently, by imaging tumor antioxidant capacity before membrane detachment, it may be possible to determine the metastatic potential of primary tumors.

\section{CONCLUSION}

The spatiotemporal assessment of the tumor redox microenvironment in vivo has the potential to inform cancer progression, therapeutic response, and metastatic potential. The preclinical development of noninvasive MRI and PET imaging agents is set to revolutionize our understanding of these dynamic processes, complementing the existing arsenal of ROS-sensing fluorophores. Clinical validation of the existing imaging agents, however, is still to be performed, along with the assessment of their prognostic utility. Additional redox-active probes are also required whose tumor retention is sensitive to the balance between ROS and the antioxidant response, rather than simple turn-on signal. With ${ }^{18} \mathrm{~F}$ FSPG already trialed in patients, there is a reasonable expectation that the first mechanistic clinical studies with this radiotracer will soon be performed.

\section{DISCLOSURE}

This research was funded in whole, or in part, by the Wellcome Trust (senior research fellowship 220221/Z/20/Z to Timothy Witney and Wellcome/EPSRC Centre for Medical Engineering grant WT203148/Z/16/Z to Hannah Greenwood). No other potential conflict of interest relevant to this article was reported.

\section{ACKNOWLEDGMENT}

We gratefully acknowledge Jim Strommer for creating Figure 1A.

\section{REFERENCES}

1. Faubert B, Solmonson A, DeBerardinis RJ. Metabolic reprogramming and cancer progression. Science. 2020;368:eaaw5473.

2. Potter M, Newport E, Morten KJ. The Warburg effect: 80 years on. Biochem Soc Trans. 2016;44:1499-1505.

3. DeBerardinis RJ, Chandel NS. We need to talk about the Warburg effect. Nat Metab. 2020;2:127-129.

4. Perillo B, Di Donato M, Pezone A, et al. ROS in cancer therapy: the bright side of the moon. Exp Mol Med. 2020;52:192-203.

5. Weinberg F, Ramnath N, Nagrath D. Reactive oxygen species in the tumor microenvironment: an overview. Cancers (Basel). 2019;11:1191. 
6. Ladner C, Ehninger G, Gey KF, Clemens MR. Effect of etoposide (VP16-213) on lipid peroxidation and antioxidant status in a high-dose radiochemotherapy regimen. Cancer Chemother Pharmacol. 1989;25:210-212.

7. Gorrini C, Harris IS, Mak TW. Modulation of oxidative stress as an anticancer strategy. Nat Rev Drug Discov. 2013;12:931-947.

8. Meister A. Glutathione metabolism. Methods Enzymol. 1995;251:3-7.

9. Espinoza EM, Røise JJ, Li I-C, Das R, Murthy N. Advances in imaging reactive oxygen species. J Nucl Med. 2021;62:457-461.

10. Kundu K, Knight SF, Willett N, Lee S, Taylor WR, Murthy N. Hydrocyanines: a class of fluorescent sensors that can image reactive oxygen species in cell culture, tissue, and in vivo. Angew Chem Int Ed Engl. 2009;48:299-303.

11. Johnson ID, Spence MTZ. The Molecular Probes Handbook: A Guide to Fluorescent Probes and Labeling Technologies. 11th ed. Molecular Probes; 2010.

12. Battisti V, Maders LD, Bagatini MD, et al. Measurement of oxidative stress and antioxidant status in acute lymphoblastic leukemia patients. Clin Biochem. 2008; 41:511-518.

13. Chu W, Chepetan A, Zhou D, et al. Development of a PET radiotracer for noninvasive imaging of the reactive oxygen species, superoxide, in vivo. Org Biomol Chem. 2014;12:4421-4431.

14. Al-Karmi S, Albu SA, Vito A, et al. Preparation of an ${ }^{18} \mathrm{~F}$-labeled hydrocyanine dye as a multimodal probe for reactive oxygen species. Chemistry. 2017;23:254 258.

15. Sivapackiam J, Liao F, Zhou D, et al. Galuminox: preclinical validation of a novel PET tracer for non-invasive imaging of oxidative stress in vivo. Redox Biol. 2020; 37:101690.

16. Solingapuram Sai KK, Bashetti N, Chen X, et al. Initial biological evaluations of ${ }^{18} \mathrm{~F}-\mathrm{KS} 1$, a novel ascorbate derivative to image oxidative stress in cancer. EJNMMI Res. 2019;9:43.

17. Lewerenz J, Hewett SJ, Huang Y, et al. The cystine/glutamate antiporter system $\mathrm{x}_{\mathrm{c}}{ }^{-}$in health and disease: from molecular mechanisms to novel therapeutic opportunities. Antioxid Redox Signal. 2013;18:522-555.

18. Koglin N, Mueller A, Berndt M, et al. Specific PET imaging of $\mathrm{x}_{\mathrm{C}}{ }^{-}$transporter activity using a ${ }^{18} \mathrm{~F}$-labeled glutamate derivative reveals a dominant pathway in tumor metabolism. Clin Cancer Res. 2011;17:6000-6011.

19. Webster JM, Morton CA, Johnson BF, et al. Functional imaging of oxidative stress with a novel PET imaging agent, ${ }^{18} \mathrm{~F}-5$-fluoro-L-aminosuberic acid. $J$ Nucl Med. 2014;55:657-664

20. Beinat C, Gowrishankar G, Shen B, et al. The characterization of ${ }^{18} \mathrm{~F}-\mathrm{hGTS} 13$ for molecular imaging of $\mathrm{x}_{\mathrm{c}}{ }^{-}$transporter activity with PET. J Nucl Med. 2019;60: 1812-1817.

21. McCormick PN, Greenwood HE, Glaser M, et al. Assessment of tumor redox status through (S)-4-(3-[ $\left.{ }^{18} \mathrm{~F}\right]$ fluoropropyl)-L-glutamic acid PET imaging of system $\mathrm{x}_{\mathrm{c}}{ }^{-}$activity. Cancer Res. 2019;79:853-863.

22. Marcato P, Dean CA, Giacomantonio CA, Lee PW. Aldehyde dehydrogenase: its role as a cancer stem cell marker comes down to the specific isoform. Cell Cycle. 2011;10:1378-1384.
23. Pereira R, Sanghera C, Greenwood HE, et al. Mapping aldehyde dehydrogenase $1 \mathrm{~A} 1$ activity using an $\left[{ }^{18} \mathrm{~F}\right]$ substrate-based approach. Chemistry. 2019;25:23452351.

24. Kirby A, Suchý M, Brouwer A, Shuhendler A. Mapping aldehydic load in vivo by positron emission tomography with $\left[{ }^{18}\right.$ F $]$ NA3BF3. Chem Commun (Camb). 2019; 55:5371-5374.

25. Hyodo F, Soule BP, Matsumoto K, et al. Assessment of tissue redox status using metabolic responsive contrast agents and magnetic resonance imaging. $J$ Pharm Pharmacol. 2008;60:1049-1060.

26. Gale EM, Mukherjee S, Liu C, Loving GS, Caravan P. Structure-redox-relaxivity relationships for redox responsive manganese-based magnetic resonance imaging probes. Inorg Chem. 2014;53:10748-10761.

27. Wang H, Jordan VC, Ramsay IA, et al. Molecular magnetic resonance imaging using a redox-active iron complex. J Am Chem Soc. 2019;141:5916-5925.

28. Ardenkjær-Larsen JH, Fridlund B, Gram A, et al. Increase in signal-to-noise ratio of $>10,000$ times in liquid-state NMR. Proc Natl Acad Sci USA. 2003;100: 10158-10163.

29. Rodrigues TB, Serrao EM, Kennedy BWC, Hu D-E, Kettunen MI, Brindle KM. Magnetic resonance imaging of tumor glycolysis using hyperpolarized ${ }^{13} \mathrm{C}$-labeled glucose. Nat Med. 2014;20:93-97.

30. Bohndiek SE, Kettunen MI, Hu D-e, et al. Hyperpolarized $\left[1-{ }^{13} \mathrm{C}\right]$-ascorbic and dehydroascorbic acid: vitamin $\mathrm{C}$ as a probe for imaging redox status in vivo. $\mathrm{J} \mathrm{Am}$ Chem Soc. 2011;133:11795-11801.

31. Timm KN, Hu DE, Williams M, et al. Assessing oxidative stress in tumors by measuring the rate of hyperpolarized $\left[1-{ }^{13} \mathrm{C}\right]$ dehydroascorbic acid reduction using ${ }^{13} \mathrm{C}$ magnetic resonance spectroscopy. J Biol Chem. 2017;292:1737-1748.

32. Keshari KR, Kurhanewicz J, Bok R, Larson PEZ, Vigneron DB, Wilson DM. Hyperpolarized ${ }^{13} \mathrm{C}$ dehydroascorbate as an endogenous redox sensor for in vivo metabolic imaging. Proc Natl Acad Sci USA. 2011;108:18606-18611.

33. D'Autréaux B, Toledano MB. ROS as signalling molecules: mechanisms that generate specificity in ROS homeostasis. Nat Rev Mol Cell Biol. 2007;8:813-824.

34. Gammon ST, Engel BJ, Gores GJ, Cressman E, Piwnica-Worms D, Millward SW. Mistiming death: modeling the time-domain variability of tumor apoptosis and implications for molecular imaging of cell death. Mol Imaging Biol. 2020;22: 1310-1323.

35. Stockwell BR, Jiang X. The chemistry and biology of ferroptosis. Cell Chem Biol. 2020;27:365-375.

36. Balendiran GK, Dabur R, Fraser D. The role of glutathione in cancer. Cell Biochem Funct. 2004;22:343-352.

37. Greenwood HE, McCormick P, Gendron T, et al. Measurement of tumor antioxidant capacity and prediction of chemotherapy resistance in preclinical models of ovarian cancer by positron emission tomography. Clin Cancer Res. 2019;25:2471-2482.

38. Paoli P, Giannoni E, Chiarugi P. Anoikis molecular pathways and its role in cancer progression. Biochim Biophys Acta. 2013;1833:3481-3498.

39. Schafer ZT, Grassian AR, Song L, et al. Antioxidant and oncogene rescue of metabolic defects caused by loss of matrix attachment. Nature. 2009;461:109-113 